\title{
Osteogenic fate of hypertrophic chondrocytes
}

\author{
Cell Research (2014) 24:1266-1269. doi:10.1038/cr.2014.111; published online 22 August 2014
}

\section{Dear Editor,}

Endochondral bone formation is largely dependent on cartilage lineage cells. The chondrocytes in growth plates continuously undergo a sequential process from proliferation to terminal hypertrophic differentiation [1]. Once differentiated, hypertrophic chondrocytes elicit multiple functions such as determining bone length, inducing osteogenesis as well as directing bone mineralization, and eventually disappear at the chondro-osseous junction. The fate of the terminally differentiated hypertrophic chondrocytes is conceptually important for understanding its role in endochondral bone formation. It has been debated for decades whether the terminally differentiated hypertrophic chondrocytes die by apoptosis or undergo osteogenic transdifferentiation, however, clear in vivo evidence is lacking [2]. Here, through lineage tracing, we provide the first in vivo evidence that the terminally differentiated hypertrophic chondrocytes are a potent source of osteoblasts, and retain multi-lineage differentiation potential.

First, we generated a novel CollOal ${ }^{\text {int } 2}$-Cre transgenic mouse strain in which Cre expression was under the control of an $8.2 \mathrm{~kb}$ promoter and a $3.2 \mathrm{~kb} 2^{\text {nd }}$ intron of the mouse type X collagen gene (CollOal), a specific marker for hypertrophic chondrocytes (Figure 1A). In situ hybridization analysis of CollOal $1^{\text {int }}$-Cre transgenic mice showed that $\mathrm{Cre}^{+}$cells were exclusively restricted to the hypertrophic zone, but not detectable in the metaphysis and bone marrow cavity from embryonic day 14.5 (E14.5) to postnatal day 10 (P10) (Figure 1A and Supplementary information, Figure S1A, S1B). Furthermore, double staining showed that all $\mathrm{Cre}^{+}$cells co-expressed Col10al, but not Collal (a marker for osteoblasts), endomucin (a marker for endothelial cells) or perilipin (a marker for adipocytes) (Supplementary information, Figure S1A, $\mathrm{S} 1 \mathrm{~B})$. In addition, RT-PCR detected $C r e-h G H$ mRNA in growth plate cartilage, but not in bone marrow or cortical bone of Col10a $1^{\text {int2 }}$-Cre transgenic mice (Supplementary information, Figure S1C). These data demonstrate that this Colloal $1^{\text {int2 }}$-Cre strain is specific for hypertrophic chondrocytes.

To track the in vivo fate and spatial distribution of hy- pertrophic chondrocytes, we generated double transgenic mice harboring Col10a $1^{\text {int2 }}$-Cre and floxed ROSA26-loxPstop-loxP-EYFP (herein ROSA ${ }^{E Y F P}$ ) reporter alleles [3]. Cre-mediated recombination within the reporter locus activates YFP expression and thus indelibly marks the recombined cell and its progeny for their entire lifespan. YFP immunostaining showed that $\mathrm{YFP}^{+}$chondrocytes occupied the lower hypertrophic zone in CollOal ${ }^{\text {int }}$ Cre; ROSA ${ }^{E Y F P}$ mice (Figure 1B). Unexpectedly, $\mathrm{YFP}^{+}$ cells were also localized throughout the metaphysis and bone marrow cavity beneath the growth plate where no $\mathrm{Cre}^{+}$cells resided (Figure 1B and Supplementary information, Figure S1D). At the age of 8 months, $\mathrm{YFP}^{+}$cells were still visible in metaphyseal, trabecular and cortical bones (Supplementary information, Figure S1D). This result indicates that beneath the growth plate cartilage, there are a considerable number of cells originated from hypertrophic chondrocytes.

To further strengthen this observation, we employed an inducible Col2al-Cre ${ }^{E R T}$ mouse line where the transgene expression is targeted to committed chondrocytes [4]. We first confirmed the restricted expression of Col2al$C r e^{E R T}$ in growth plate cartilage from $\mathrm{P} 3$ to $\mathrm{P} 15$ by in situ hybridization analysis (Supplementary information, Figure S1E). After a single dose of tamoxifen administration at P5, Col2al-Cre ${ }^{E R T} ; R O S A^{E Y F P}$ mice gradually displayed unifying clonal columns of $\mathrm{YFP}^{+}$cells (Figure 1C). On day 10 after tamoxifen induction, some $\mathrm{YFP}^{+}$columns clearly extended from proliferating chondrocyte zone to the metaphysis (Figure 1D), implying that the progenies of chondrocytes translocated to metaphysis. Furthermore, we exploited the multicolor Confetti reporter system, a powerful tool for clonal analysis, in which Col2a1-Cre $e^{E R T}$ mediated recombination randomly activates one of its four reporters (Supplementary information, Figure S1F) [5]. After 5 days following tamoxifen treatment at P5, we observed the labeled clonal columns with representation of the different colors in the growth plate of Col2a1$C r e^{E R T} ; R_{O S A} A^{\text {Confetti }}$ mice (Supplementary information, Figure S1G). On day 10 after tamoxifen exposure, some chondrocyte clones labeled by the same color elongated into metaphysis underneath the growth plate (Figure 1E and Supplementary information, Figure $\mathrm{S} 1 \mathrm{H}$ ), similar 
A

Col10a1 promoter (8.2 kb) -Cre-hGH polyA - intron 2

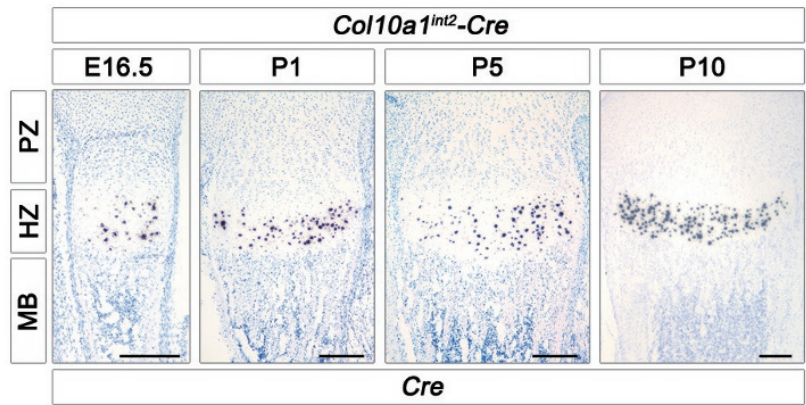

C

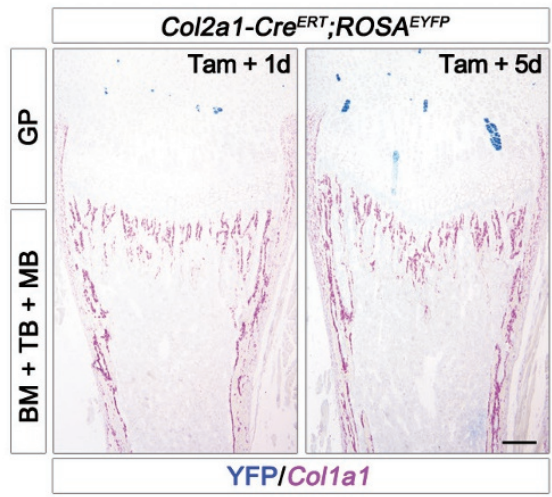

B

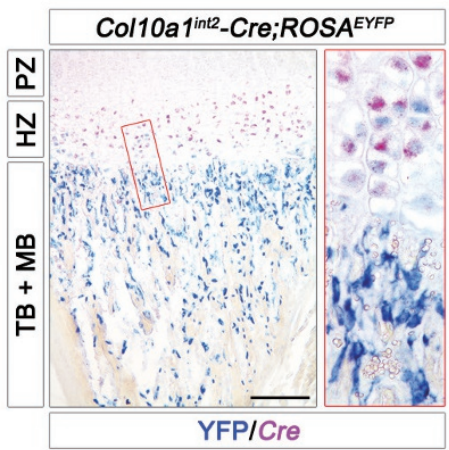

E

F
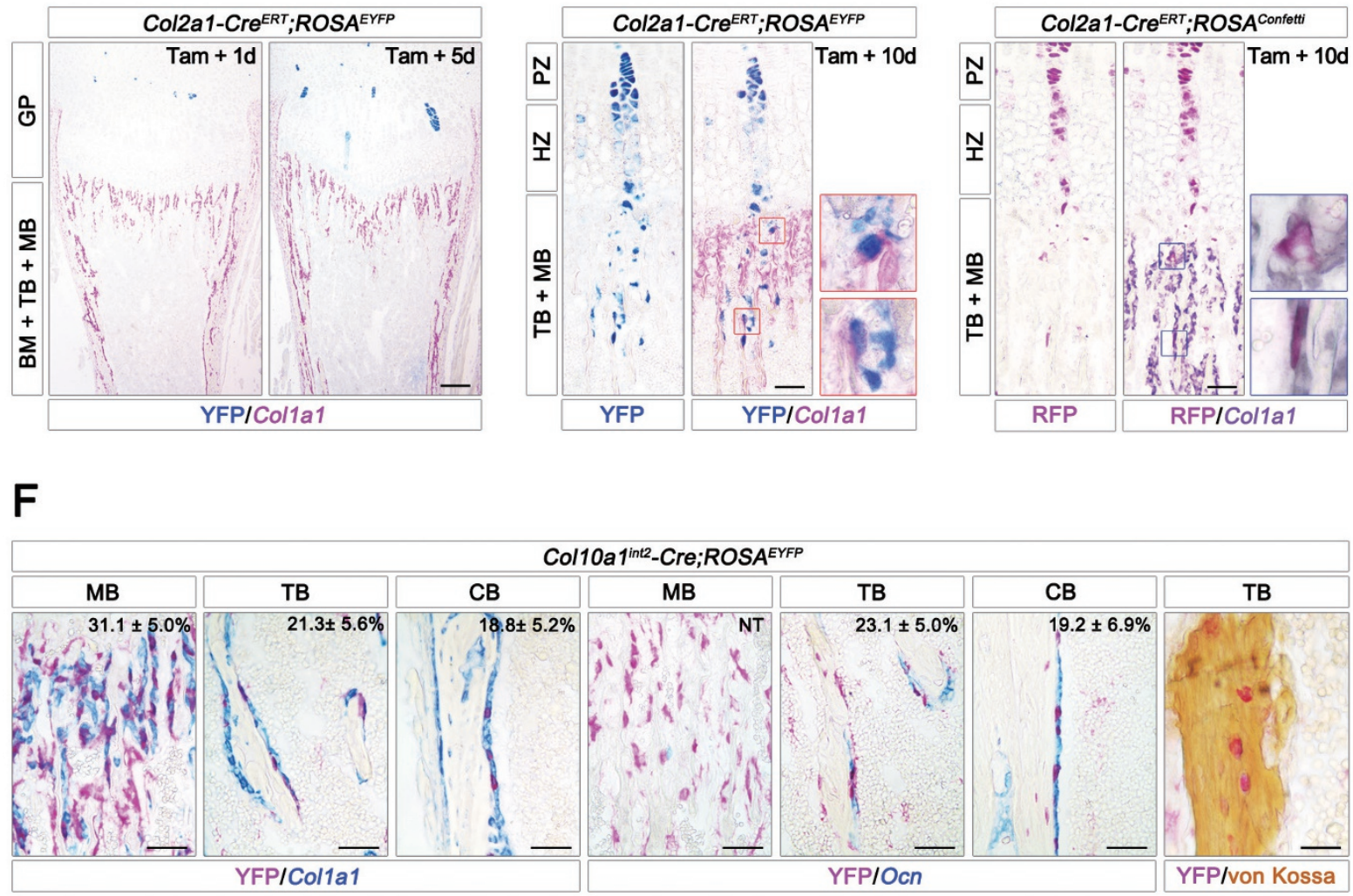

G

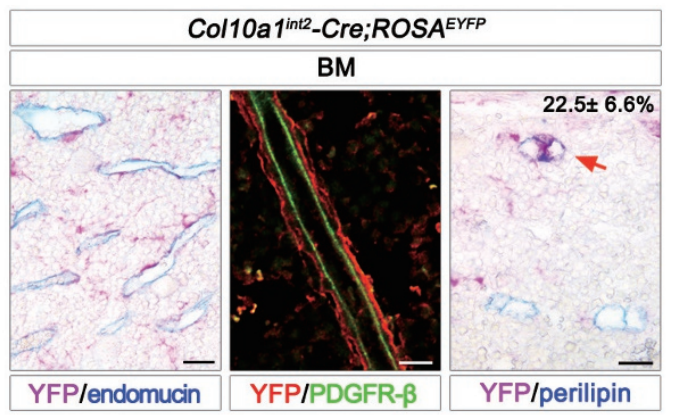

$\mathrm{H}$

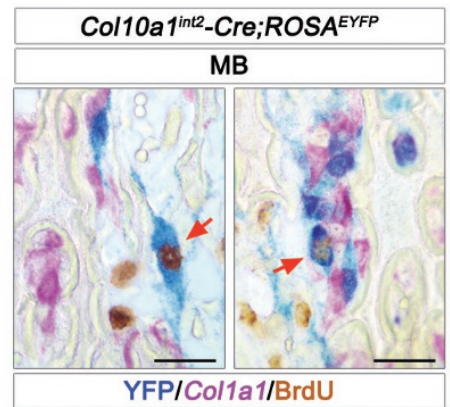


to the findings from the Col2al-Cre ${ }^{E R T} ; \operatorname{ROSA} A^{E Y F P}$ mice. Taken together, these data strongly indicate that the progenies of chondrocytes entering into metaphysis and bone marrow cavity are still alive and active, raising a possibility that hypertrophic chondrocytes may give rise to other cell types.

To determine the $\mathrm{YFP}^{+}$cell types within metaphysis and bone marrow cavity of CollOa1 $1^{\text {int }}-\mathrm{Cre}$; ROSA $A^{E Y F P}$ mice, markers for osteogenic lineage cells and stromal cells were used. At the age of 10 days, 20 days and 8 months, some $\mathrm{YFP}^{+}$cells presented as osteogenic lineage cells, as evidenced by co-expression of endogenous transcripts of Collal, osteocalcin (Ocn) and bone sialoprotein (Bsp), markers for osteoblastic differentiation (Figure $1 \mathrm{~F}$ and Supplementary information, Figure S1D). Consistent with this, double labeled $\mathrm{YFP}^{+} \mathrm{Collal}^{+}$cells were also observed in Col2al-Cre ${ }^{E R T} ; R_{O S A} A^{E Y F P}$ mice and Co$12 a 1-C r e^{E R T} ; R O S A^{\text {Confetti }}$ mice (Figure 1D-1E and Supplementary information, Figure S1H). Quantification of the $\mathrm{YFP}^{+} \mathrm{Colla}^{+}$proportion in total Colla1 ${ }^{+}$osteoblasts in 20-day-old Col10a $1^{\text {int2 }}$-Cre;ROSA ${ }^{\text {EYFP }}$ mice revealed that $\mathrm{YFP}^{+}$cells accounted for approximately $31.1 \%, 21.3 \%$ and $18.8 \%$ of Colla1 ${ }^{+}$cells in metaphyseal, trabecular and cortical bones, respectively (Figure 1F). Von Kossa staining suggested that at the age of 2 months, the $\mathrm{YFP}^{+}$ cells entrapped within the bone were osteocytes (Figure 1F). These data strongly demonstrate that hypertrophic chondrocytes directly give rise to osteoblasts and osteocytes during endochondral bone formation.

In addition to the osteogenic fate, hypertrophic chon- drocytes exhibited multiple destinies. In the bone marrow of Colloal $1^{\text {int }}-\mathrm{Cre} ; \mathrm{ROSA}^{E Y F P}$ mice, some $\mathrm{YFP}^{+}$cells displayed specific perivascular localization, as evidenced by their enfolding of the endomucin ${ }^{+}$vascular endothelial cells and PDGFR- $\beta^{+}$pericytes (Figure $1 \mathrm{G}$ and Supplementary information, Figure S1D). Moreover, about $22.5 \%$ of the adipocytes labeled by perilipin co-localized with YFP staining (Figure $1 \mathrm{G}$, red arrow), representing another fate of hypertrophic chondrocytes. Importantly, $\mathrm{YFP}^{+}$cells in metaphysis showed proliferative ability, as evidenced by BrdU incorporation (Figure $1 \mathrm{H}$, red arrow). As expected, some $\mathrm{YFP}^{+} \mathrm{Colla1}^{+}$osteoblasts were BrdUpositive (Figure $1 \mathrm{H}$, red arrow).

This study reveals the in vivo mutilpotential cell fates of hypertrophic chondrocytes. Supporting the transdifferentiation hypothesis based on an in vitro experiment 20 years ago [6], our results demonstrate that the chondrocyte progeny have the capacity to turn into osteoblasts and osteocytes in the developing bone, providing new insight into the roles of hypertrophic chondrocytes in coupling chondrogenesis and osteogenesis. It is well accepted that by the production of regulatory factors, hypertrophic chondrocytes instruct adjacent perichondrial cells to differentiate into osteoblasts. Unexpectedly, the chondrocyte-to-osteoblast transition verified in our study also points to a direct role of hypertrophic chondrocytes in osteogenesis. Our observations differ from a previous lineage-tracing study that also used the Col2al-Cre $e^{E R T}$ transgenic line, which showed that chondrocytes do not contribute to the embryonic osteoblast pool [7]. Besides

Figure 1 Multipotential fates of hypertrophic chondrocyte. (A) The transgenic vector contained an $8.2 \mathrm{~kb}$ promoter of mouse type $\mathrm{X}$ collagen (Col10a1), Cre cDNA, human growth hormone $(h G H)$ polyadenylation signal and a $3.2 \mathrm{~kb} 2^{\text {nd }}$ intron of Col10a1. Isotopic in situ hybridization assay was used to detect the transgenic Cre mRNA in tibia proximal growth plate from Col10a $1^{\text {int2 }}$-Cre transgenic mice at E16.5, P1, P5 and P10. (B) Double staining of YFP (blue) by immunohistochemistry (IHC) and Cre mRNA (fuchsia) by in situ hybridization in 10-day-old Col10a $1^{\text {int2 }}$-Cre; ROSA ${ }^{E Y F P}$ tibia. The right panel shows the high-magnification image of the area boxed in red on the left. (C) After 1 and 5 days following tamoxifen administration $(50 \mathrm{mg} / \mathrm{kg})$ at P5, Col2a1-Cre ${ }^{E R T} ; R O S A^{E Y F P}$ tibia was stained by YFP (blue) and Col1a1 (fuchsia) antibodies. The clonal columns of YFP $^{+}$cells were detectable in Col2a1-Cre ${ }^{\text {ERT }}$;ROSA ${ }^{\text {EYFP }}$ chondrocyte zone. (D) After 10 days following tamoxifen administration at P5, Col2a1-Cre ${ }^{E R T} ; R O S A^{E Y F P}$ tibia was stained by YFP (blue) antibody, showing a column of YFP ${ }^{+}$cells entering into the metaphysis. IHC double staining of YFP (blue) and type I collagen (Col1a1) (fuchsia) showed that some $\mathrm{YFP}^{+}$cells underneath the growth plate were labeled with Col1a1 (red frames, high-magnification images of areas boxed on the left). (E) After 10 days following tamoxifen administration $(50 \mathrm{mg} / \mathrm{kg})$ at P5, Col2a1-Cre ${ }^{E R T} ; R O S A^{\text {Confetti }}$ tibia was stained by RFP (fuchsia) antibody. Double staining of RFP (fuchsia) by IHC and Col1a1 (purple grey) mRNA by in situ hybridization showed that some $\mathrm{RFP}^{+}$cells in metaphysis were Col1a1-positive (red frames, high-magnification images of areas boxed on the left). (F) Double staining of YFP (fuchsia) by IHC and the transcripts of Col1a1 or osteocalcin (Ocn) (blue) by in situ hybridization in metaphyseal, trabecular and cortical bones from 20-day-old Col10a $1^{\text {int2 }}-\mathrm{Cre} ; \mathrm{ROSA}^{\text {EYFP }}$ tibia. The percentages of $\mathrm{YFP}^{+} \mathrm{Col}_{1} \mathrm{a}^{+}{ }^{+}$and $_{\mathrm{YFP}} \mathrm{OCn}^{+}$ osteogenic cells in total Col1 $1^{+}$and $\mathrm{Ocn}^{+}$cells from 3 images per mouse $(n=3)$ are shown in the top-right corner. Von Kossa staining showed that some YFP ${ }^{+}$cells (fuchsia) within the mineralized bone were osteocytes. (G) In 20-day-old Col10a $1^{\text {int2 }}$-Cre;ROSA ${ }^{\text {EYFP }}$ bone marrow (BM), double immunostaining (left) of YFP (fuchsia) and endomucin (blue, marker for endothelial cells) as well as double immunofluorescence analysis (middle) using YFP (red) and PDGFR- $\beta$ (green, marker for pericytes) antibodies indicated that some $\mathrm{YFP}^{+}$cells were adjacent to the endothelial cells and vascular pericytes. Double immunostaining (right) of YFP (fuchsia) and perilipin (blue, marker for adipocytes) showed that some $\mathrm{YFP}^{+}$cells were adipocytes (red arrow). (H) Triple staining of YFP (blue) and BrdU (brown) by IHC along with Col1a1 mRNA (fuchsia) by in situ hybridization in 20-day-old Col10a $1^{\text {int2 }}$-Cre; $R O S A^{\text {EYFP }}$ metaphyseal bones (MB). PZ, proliferation zone; HZ, hypertrophic zone; MB, metaphyseal bone; TB, trabecular bone; BM, bone marrow. Scale bars are $200 \mu \mathrm{m}$ (A-C), $50 \mu \mathrm{m}$ (D-F) and $25 \mu \mathrm{m}(\mathbf{G}, \mathbf{H})$. 
the different transgenic strains and induction time windows, the apparent discrepancy is probably due to the relatively short time frame of the previous study, which may have missed the ultimate fate of chondrocytes. Recent lineage tracing studies have confirmed that perichondrial osteoblast precursors and bone marrow mesenchymal stem cells contribute to trabecular osteoblasts in the developing and adult skeleton, respectively [7, 8]. Here, our findings demonstrate that growth plate chondrocytes provide a new potent source of osteoblasts during bone growth. We also reveal that hypertrophic chondrocytes adopt perivascular cell and adipocyte fates in addition to the osteogenic fate. Given that endochondral ossification is required for hematopoietic stem cell niche formation [9], the chondrocyte progeny might not only benefit bone formation but also support hematopoiesis.

\section{Acknowledgments}

This work was supported by the Chinese National Key Program on Basic Research (2012CB945103, 2012CB966904, 2011CB504202), and National Natural Science Foundation of China (31030040, 81241062, 31171249, 81272702). We thank Xizhi Guo for ROSA26 ${ }^{E Y F P}$ reporter mice and Peng Li for perilipin antibody.

Guan Yang ${ }^{1, *}$, Liang $\mathrm{Zhu}^{1, *}$, Ning Hou ${ }^{1}$, Yu Lan ${ }^{1}$, Xi-Mei $\mathrm{Wu}^{2}$, Bin Zhou ${ }^{3}$, Yan Teng ${ }^{1}$, Xiao Yang ${ }^{1}$

${ }^{1}$ State Key Laboratory of Proteomics, Genetic Laboratory of Development and Diseases, Institute of Biotechnology, 20 Dongdajie, Beijing 100071;
${ }^{2}$ Department of Pharmacology, School of Medicine, Zhejiang University, Hangzhou, Zhejiang 310058; ${ }^{3}$ Key Laboratory of Nutrition and Metabolism, Institute for Nutritional Sciences, Shanghai Institutes for Biological Sciences, Graduate School of the Chinese Academy of Sciences, Chinese Academy of Sciences, Shanghai 200031, China

*These two authors contributed equally to this work.

Correspondence: Guan Yang ${ }^{\mathrm{a}}$, Yan Teng ${ }^{\mathrm{b}}$, Xiao Yang ${ }^{\mathrm{c}}$

${ }^{\mathrm{a}}$ Tel/Fax: 86-10-63895937

E-mail: yogopop3@163.com

${ }^{\mathrm{b}}$ Tel/Fax: 86-10-63895937

E-mail: tengyan0919@163.com

${ }^{\mathrm{c}}$ Tel/Fax: 86-10-63895937

E-mail: yangx@bmi.ac.cn

\section{References}

1 Kronenberg HM. Nature 2003; 423:332-336.

2 Shapiro IM, Adams CS, Freeman T, et al. Birth Defects Res C Embryo Today 2005; 75:330-339.

3 Srinivas S, Watanabe T, Lin CS, et al. BMC Dev Biol 2001; 1:4.

4 Hilton MJ, Tu X, Long F. Dev Biol 2007; 308:93-105.

5 Snippert HJ, van der Flier LG, Sato T, et al. Cell 2010; 143:134-144.

6 Descalzi Cancedda F, Gentili C, Manduca P, et al. J Cell Biol 1992; 117:427-435.

7 Maes C, Kobayashi T, Selig MK, et al. Dev Cell 2010; 19:329-344.

8 Park D, Spencer JA, Koh BI, et al. Cell Stem Cell 2012; 10:259-272.

9 Chan CK, Chen CC, Luppen CA, et al. Nature 2009; 457:490-494.

(Supplementary information is linked to the online version of the paper on the Cell Research website.)

cc)(i) (s) $\Theta$ This work is licensed under the Creative Commons Attribution-NonCommercial-No Derivative Works 3.0 Unported License. To view a copy of this license, visit http:// creativecommons.org/licenses/by-nc-nd/3.0 